\title{
ENUMERATION OF THE ARALIACEAE COLLECTED BY L. J. BRASS IN NEW GUINEA ${ }^{1}$
}

\section{H. Harms}

Boerlagiodendron forsan aff. B. Ledermannii Harms in Bot. Jahrb. 56: 383 (1921).

Fly River, 528 mile Camp, $80 \mathrm{~m}$. alt. Common along edge of forest on muddy banks of river. Sparsely branched tree 2-4 m.; inflorescence purple. May, 1936; L. J. Brass 6794.

Boerlagiodendron aff. B. stenolobum Harms, l. c. 382.

Palmer River, 2 miles below junction Black River, muddy banks of sluggish backwater creeks. Sparsely branched shrub 2-3 m.; inflorescence purple. June, 1936; L. J. Brass 7056.

Specimen lobis folii latioribus differt.

\section{Boerlagiodendron (folium).}

Fly River, 528 mile Camp, $80 \mathrm{~m}$. alt. Common along edge of forest on muddy banks of river. Sparsely branched tree 2-4 m.; inflorescence purple. May, 1936; L. J. Brass 6794.

Schefflera macrostachya (Benth.) Harms; cf. Harms in Bot. Jahrb. 56: 389 (1921).

Rona, Central Division, $450 \mathrm{~m}$. alt. Common on or about rocks outside rain forest in Laloki River gorge. Tree to $12 \mathrm{~m}$. tall, few thick branches, erect or nearly erect, at apex large leaves; petiole near $1 \mathrm{~m}$. long; persistent stipule; flowers red, in stout terminal panicle $20-30 \mathrm{~cm}$., erect above leaves. Feb., 1936; L. J. Brass 6209.- Upper Wassi Kussa River (left branch), Western Division; rain forest, epiphyte on riverbank trees. Tree 6-8 m., branches of inflorescence spreading when in fruit; flowers red; fruits black, fleshy. January, 1937; L. J. Brass 8618 .

Species in Papuasia late distributa.

Schefflera Rudolfi Harms, 1. c. 394.

Palmer River, 2 miles below Black River Junction, 100 m. alt. Sparsely branched shrub (epiphytic), found near ground in all types of rain forest. April, 1936; L. J. Brass 6891.- Same locality. Unbranched

${ }^{1}$ Botanical Results of the Archbold Expeditions. 
shrub, $1.5 \mathrm{~m}$. h.; terrestrial in forest undergrowth; fruit red. June, 1936; L. J. Brass 7055 .

Specimina pedunculis longioribus a typo diversa.

Schefflera venulosa (W. \& A.) Harms; cf. Harms, l. c. 406.

Lower Fly River, east bank opposite Sturt Island; rain forest. High climbing liane, common on ridges; panicle yellow; ripe fruit red. October, 1936; L. J. Brass 8040.

Schefflera Versteegii Harms, 1. c. 403.

Palmer River, 2 miles below Black River Junction, 100 m. alt. Arborescent epiphyte in ridge forest; stem unbranched; panicle terminal, $40 \mathrm{~cm}$. long., with many radial branches; flowers green. July, 1936; L. J. Brass 7296.

Schefflera Brassii Harms, sp. nov.

Frutex epiphyticus magnus; folia magna digitata, vagina lata crassa setis laciniiformibus obsita, petiolo in folio unico suppetante glabro 55 $\mathrm{cm}$. longo, apice nodoso-incrassato; foliola 5 (probaliter plura, forsan 7-9), oblongo-oblanceolata vel anguste oblonga, basi angustata rotundata vel obtusa, apice acuminata, chartacea, glabra, nervis lateralibus utrinque circ. 7-11 cum costa subtus prominulis inter se spatio lato distantibus; panicula terminalis, ampla, pedunculo puberulo, bracteis lanceolatis setulis vel setis laciniiformibus obsitis, rhachi puberula $20 \mathrm{~cm}$. longa, paniculas secundarias complures (8-10) racemiformes umbelluligeras $12-25 \mathrm{~cm}$. longas gerente, rhachibus tenuibus puberulis; umbellulae racemose dispositae numerosae, parvae, pedunculis puberulis $6-7 \mathrm{~mm}$. longis, ad basim florum parce pilosis, 7-10-florae, pedicellis 3-4 $\mathrm{mm}$. longis, cum ovario fere $1.5 \mathrm{~mm}$. longo glabris; corolla in specimine delapsa; stamina 5; discus leviter elevatus, stylis obsoletis.

Papua: Middle Fly River, Lake Daviumbu, September, 1936; L. J. Brass 7746; large epiphytic shrub, common in rain forests, flowers white.

Species floribus minimis in umbellulas parvas vel rhachin elongatam dispositis distincta.

Schefflera fimbriata (F. Muell.) Harms; cf. Harms in Bot. Jahrb. 56: 402 (1921). - Heptapleurum fimbriatum F. Muell. (1877).

Fly River, 528 mile Camp, $80 \mathrm{~m}$. alt. Common epiphytic shrub in river flood-bank forest. May, 1936; L. J. Brass 6786.- Palmer River, 2 miles below Black River Junction. Unbranched shrub $1 \mathrm{~m}$. June, 1936; L. J. Brass 6912.- Same locality, $100 \mathrm{~m}$. alt. Common low shrub on river-banks; fruit purple-red. July, 1936; L. J. Brass 7239.- 
Same locality. Epiphytic, 2-3 m. above ground level on river-bank trees; unbranched shrub, $1.5 \mathrm{~m}$; flowers white. July, 1936; L. J. Brass 7261.

Flores haud in spicas dispositi (ut prius putavi), sed in umbellulas capituliformes ad rhachin elongatam subsessiles.

Polyscias Caroli Harms in Bot. Jahrb. 56: 411 (1921).

Lake Daviumbu, Middle Fly River; edge of light rain forest. Sparsely branched shrub, 1-2 m. h.; flowers white. September, 1936; L. J. Brass 7922. - Gaima, Lower Fly River (east bank). Common in outskirts of rain forest. Shrub, 1-2 m.; branched or not branched. September, 1936; L. J. Brass 8372. - Tarara, Wassi Kussa River, Western Division. Common in inferior rain forest. Shrub 1-1.5 m.; ripe fruit, soft, black. January, 1937 ; L. J. Brass 8740.

Polyscias elegans (C. Moore \& F. Muell.) Harms.

Tarara, Wassi Kussa River, Western Division. Tree 7-9 m. common in rain forest substage; inflorescence purple, anthers yellow. Terminal panicle about $40 \mathrm{~cm}$. long. January, 1937; L. J. Brass 8656, 8748.

Polyscias Branderhorstii Harms in Bot. Jahrb. 56: 412 (1921) forsan serius cum $P$. elegans conjugenda.

\section{Anomopanax cf. A. Schlechteri Harms.}

Palmer River, 2 miles below Black River Junction, 100 m. alt. Rare in forest undergrowth; plant unbranched, $60 \mathrm{~m}$. high; flowers white. July, 1936; L. J. Brass 7322.

Anomopanax variaefolius C. T. White in Jour. Arnold Arb. 10: 256 (1929).

Fly River, Oroville Camp. Epiphyte in forest; large fleshy sparsely branched shrub; leaves 80-90 cm. long; flowers cream-colored. August, 1936 ; L. J. Brass 7409.

\section{Botanisches Museum, \\ Berlin-Dahlem.}




\section{$2 \mathrm{BHL}$ Biodiversity Heritage Library}

Harms, Hermann. 1939. "Enumeration of the Araliaceae Collected by L.J. Brass in New Guinea." Journal of the Arnold Arboretum 20(3), 321-323. https://doi.org/10.5962/p.325782.

View This Item Online: $\underline{\text { https://www.biodiversitylibrary.org/item/33596 }}$

DOI: https://doi.org/10.5962/p.325782

Permalink: https://www.biodiversitylibrary.org/partpdf/325782

\section{Holding Institution}

Missouri Botanical Garden, Peter H. Raven Library

\section{Sponsored by}

Missouri Botanical Garden

\section{Copyright \& Reuse}

Copyright Status: In copyright. Digitized with the permission of the rights holder.

Rights Holder: Arnold Arboretum of Harvard University

License: http://creativecommons.org/licenses/by-nc-sa/3.0/

Rights: https://biodiversitylibrary.org/permissions

This document was created from content at the Biodiversity Heritage Library, the world's largest open access digital library for biodiversity literature and archives. Visit BHL at https://www.biodiversitylibrary.org. 